\title{
Spin Physics with Photon Beams
}

\author{
P. Pedroni* \\ INFN, Sezione di Pavia - Pavia, Italy \\ E-mail: paolo.pedroni@pv.infn. it
}

for the A2@MAMI Collaboration

The A2 Collaboration at MAMI performs a manifold research program with real photons at the MAMI accelerator facility in Mainz (Germany) with the Crystal Ball/TAPS setup which, providing almost complete coverage in solid angle, is well suited for the detection and the identification of multi-particle final states.

In particular, the internal structure of the nucleon is studied via measurements of unpolarized cross sections and various polarization observables both in Compton scattering and meson photoproduction reactions reactions. These experiments make use of high-intensity unpolarized, linearly or circularly polarized photon beams, and unpolarized or polarized targets.

In this presentation, recent results from the Compton scattering and baryon spectroscopy programs are presented.

23rd International Spin Physics Symposium - SPIN2018 -

10-14 September, 2018

Ferrara, Italy

${ }^{*}$ Speaker. 


\section{Introduction}

The internal structure of the nucleon has been an important subject of intense study in nuclear and particle physics since many decades, both from the experimental and theoretical point of view. In particular, the precise determination of the nucleon polarizabilities and of the the baryon resonance properties gives essential constraints on all models of the nucleon structure, as it will briefly outlined in the following sections.

\subsection{Meson Photoproduction}

The excited states of the nucleon are related to the fundamental properties of the strong interaction, just like the excitation spectra of atoms reflect the properties of the electromagnetic interaction. For this reason the investigation of the excited states of the nucleon is a fundamental step towards the understanding of the nucleon structure. This investigation can be performed with the electromagnetic nucleon excitation to a resonant $N^{*}$ state and with the study of its strong decay via (multi) meson emission.

Considering the simple case of single pseudo-scalar meson (i.e., $\pi, \eta, .$.$) production off a nu-$ cleon, we can see that we have 8 possible combination of spin states. The scattering amplitude is thus described by eight complex matrix elements only 4 of which are independent [1]. In the basis of Pauli spinors the differential cross section in the CM frame is given by

$$
\frac{d \sigma}{d \Omega}=\frac{q}{k}\langle f|\mathscr{F}| i\rangle
$$

with initial state $|i\rangle$ and final state $|f\rangle$. The phase-space factor $q / k$ is given by the momentum $k$ of the incoming photon and the momentum $q$ of the produced meson, both in the CM frame. $\mathscr{F}$ can be expressed in terms of the four CGLN amplitudes $F_{1}, F_{2}, F_{3}, F_{4}$ [1] and it is possible to separate the energy dependence from the angular dependence by performing an expansion in terms of derivatives of Legendre polynomials $P_{l}$. For the $F_{1}$ amplitude, for instance, we obtain:

$$
F_{1}(W, \theta)=\sum_{l=0}^{\infty}\left[l M_{l+}(W)+E_{l+}(W)\right] P_{l+1}^{\prime}(\cos \theta)+\left[(l+1) M_{l .}(W)+E_{l-}(W)\right] P_{l-1}^{\prime}(\cos \theta)
$$

where the kinematic variables, $W$ and $\theta$, correspond to the total $\pi N$ energy and the pion emission polar angle in the c.m. frame. The coefficients, or multipoles, $E_{l \pm}$ and $M_{l \pm}$ refer to transitions into a final state with orbital angular momentum $l$ between the recoiling baryon and the produced meson, and total angular momentum $J=l \pm 1 / 2$. The $E_{l \pm}$ coefficient refer to transitions initiated by the electrical component of the photon, and the $M_{l \pm}$ coefficient to transitions initiated by the magnetic component. Thus, each multipole refers to a state with a well defined $J^{P}$, with the parity $P=(1)^{l+1}$.

With these four complex amplitudes, 16 bilinear products can be constructed, which can be acccessed by experiments involving polarization by selecting individual spin configurations of the initial state particles, by measuring the polarization in the final state, or both. They correspond to the 16 observables listed in Table 1: the unpolarized differential cross section $\sigma_{0}$, three single $(\Sigma$, $P, T)$ and 12 double polarization observables. The measurement of $\sigma_{0}$, all three single polarization 
Table 1: Overview of all 16 possible polarization observables that can be beasured in pseudoscalar meson photoproduction reactions. The unpolarized cross section is highlighted in green, the single polarization observables in blue and all double polarization in red. The observables in bracket show alternative ways on how to obtain a specific observable.

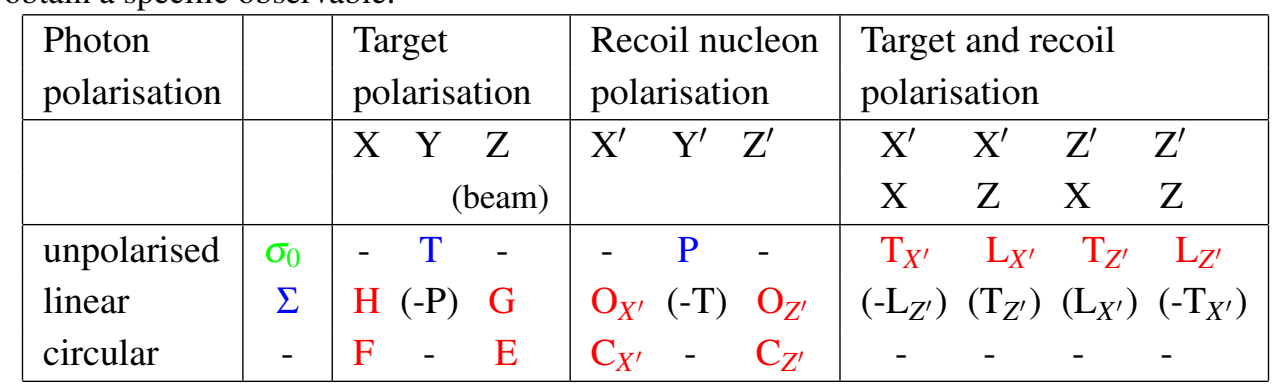

observables and four (properly-chosen) of the double polarization observables lead to an unambiguous solution for the CGLN amplitudes up to one phase [2]. This is known as the complete experiment [3].

All the different polarization observables are also an essential tool to to solve the long-standing "missing baryon resonances" problem, i.e. the fact that the number of the states predicted both by the different quark models and by the more recent LQCD calculations [4] predict a number of resonances that is is much larger than the number of states that have been actually observed so far. The advantage in using these observables is due to the fact that many of them contain interference terms between the different multipoles, making it possible to determine small, but important amplitudes in a unique way and then to disentangle the complicated nucleon resonance spectrum, which contains many broad and highly overlapping states.

Despite of their importance, there is a lack of high quality data in most of the different polarization observables, not only for the $\pi N$ final state but also from other possible resonance decay channels $(\eta N, \pi \pi N, \ldots)$. A systematic measurements of several polarization observables for different rewaction channels, both on proton and neutron is being carried out at Mainz (Germany) by the A2@MAMI collaboration with the ambitious goal to obtain the first "complete" data set. A few selected results will be given in the following.

\subsection{Compton Scattering}

Nucleon polarizabilities are fundamental structure properties which are sensitive to the internal quark dynamics of the nucleon. They can be accessed by measuring the differential cross section and singly and doubly polarized asymmetries in real Compton scattering. In addition to their obvious interest as nucleon structure observables, nucleon polarizabilities also limit the precision that can be accessed in many other areas of physics, from astrophysics to atomic physics [5].

The proton's electric and magnetic polarizabilities appear in the second order term in the Compton scattering Hamiltonian:

$$
H_{\text {eff }}^{(2)}=-4 \pi\left[\frac{1}{2}\left(\alpha_{E 1} \vec{E}^{2}+\beta_{M 1} \vec{H}^{2}\right)\right],
$$

where $\alpha_{E 1}=(11.2 \pm 0.4) 10^{4} \mathrm{fm}^{3}$ and $\beta_{M 1}=(2.5 \mp 0.4) 10^{4} \mathrm{fm}^{3}$ [6]. Despite their fundamental nature and great theoretical interest, there is still a large uncertainty in the nucleon scalar polar- 
izabilities: while $\alpha_{E 1}$ is rather well constrained by experimental data, $\beta_{M 1}$ is less certain, and the neutron data are very scarce.

The spin polarizabilities appear in the third order term of the effective interaction Hamiltonian:

$$
H_{e f f}^{(3)}=4 \pi\left[\frac{1}{2} \gamma_{E 1 E 1} \vec{\sigma} \cdot \vec{E} \times \dot{\vec{E}}+\frac{1}{2} \gamma_{M 1 M 1} \vec{\sigma} \cdot \vec{H} \times \dot{\vec{H}}-\gamma_{E 1 M 2} H_{i j} \sigma_{i} E_{j}+\gamma_{M 1 E 2} E_{i j} \sigma_{i} H_{j}\right]
$$

involving one field derivative with respect to either time or space. The four $\gamma$ factors, called the spin polarizabilities, depict the response to an excitation by an electric or magnetic dipole ( $E 1$ or $M 1)$ or quadrupole ( $E 2$ or $M 2)$ followed by a de-excitation by an electric or magnetic dipole. Unlike the scalar polarizabilities, there had been no experimental determination of the values, other than several linear combinations of them such as the forward $\left(\gamma_{0}\right)$ and backward $\left(\gamma_{\pi}\right)$ spin polarizabilities:

$$
\begin{aligned}
& \gamma_{0}=-\gamma_{E 1 E 1}-\gamma_{M 1 M 1}-\gamma_{E 1 M 2}-\gamma_{M 1 E 2} \\
& \gamma_{\pi}=-\gamma_{E 1 E 1}+\gamma_{M 1 M 1}-\gamma_{E 1 M 2}+\gamma_{M 1 E 2}
\end{aligned}
$$

for which $\gamma_{0}=(1.0 \pm 0.08) \cdot 10^{4} \mathrm{fm}^{4}[7,8]$ and $\gamma_{\pi}=(8.0 \pm 1.8) \cdot 10^{4} \mathrm{fm}^{4}$ [9].

To address this need for experimentally extracted spin polarizabilities, as well as improved scalar polarizabilities, the A2@MAMI collaboration began a program of Compton scattering experiments to investigate the proton polarizabilities. This program uses (un)polarized photon beams with both polarized and unpolarized proton targets to measure the unpolarized differential cross section as well as three asymmetries

$$
\Sigma_{2 x / z}=\frac{N_{x / z}^{R}-N_{x / z}^{L}}{P_{\gamma} P_{t}\left(N_{x / z}^{R}+N_{x / z}^{L}\right)} \quad ; \quad \Sigma_{3}=\frac{N_{\|}-N_{\perp}}{P_{\gamma}\left(N_{\|}+N_{\perp}\right)} .
$$

The first two of these asymmetries rely on a circularly polarized photon beam and a transversely or longitudinally polarized proton target, respectively, where $N_{x / z}^{R / L}$ represents the number of events with $x / z$ target polarization and $R / L$ beam helicity, $P_{\gamma}$ represents the magnitude of the beam circular polarization, and $P_{T}$ represents the magnitude of the target polarization. The third relies on a linearly polarized photon beam on an unpolarized proton target, where $N_{\| / \perp}$ represents the number of events with $\| / \perp$ beam linear polarization, and $P_{\gamma}$ represents the polarization magnitude.

\section{The A2 Experimental Setup}

The experimental setup of the A2 Collaboration is located at the Mainz-MAMI tagged photon beam facility. The photon beam is produced via bremsstrahlung of an electron beam of 1557 $\mathrm{MeV}$ provided by the MAMI accelerator, and polarised up to about $80 \%$. The photons are then tagged using the Glasgow-Mainz magnetic spectrometer, which has an energy resolution of about $4 \mathrm{MeV}$. The A2 apparatus can make use of different targets: for the masurements described in the following, a frozen-spin butanol $\left(\mathrm{C}_{4} \mathrm{H}_{9} \mathrm{OH}\right)$ [10] has been used. The $2 \mathrm{~cm}$ long target cell was located in a ${ }^{3} \mathrm{He} /{ }^{4} \mathrm{He}$ dilution refrigerator and the target nucleons were polarised via Dynamic Nuclear Polarisation (DNP) [11] to an initial polarisation degree up to about $90 \%$. During the data taking, a magnetic field of $0.68 \mathrm{~T}$ together with the $25 \mathrm{mK}$ temperature provided by the dilution 
refrigerator ensured long relaxation times (up to $2000 \mathrm{~h}$ ) before repolarisation was required. Since only the hydrogen nuclei of the butanol can be polarised, a carbon foam target was used in a dedicated beamtime to study background contributions from unpolarised carbon and oxygen nuclei.

To analyze the final state of the interaction, a nearly $4 \pi$ detector system is put in place over the target. This detector system is composed of the Crystal Ball (CB) [12] and (TAPS) [13] photon spectrometers. The CB is an array of $672 \mathrm{NaI}$ crystals, covering the azimuthal $(\phi)$ and polar $(\theta)$ angular regions from $0^{\circ}$ to $360^{\circ}$ and from $21^{\circ}$ to $159^{\circ}$, respectively.

This is coupled with a barrel of 24 plastic scintillators called the Particle Identification Detector (PID), as well as a pair of Multi-Wire Proportional Chambers (MWPCs), for charged particle identification and tracking [14]. TAPS is a vertical wall of $384 \mathrm{BaF} 2$ and $72 \mathrm{PbWO} 4$ crystals, which covers the downstream hole of the CB. It has an additional wall of plastic scintillators for charged particle identification. Finally, there is a possibility to measure the polarization of recoil nucleons with a polarimeter (a graphite cylinder) sandwiched between the PID barrel and the MWPCs. This system is shown in Fig.1.

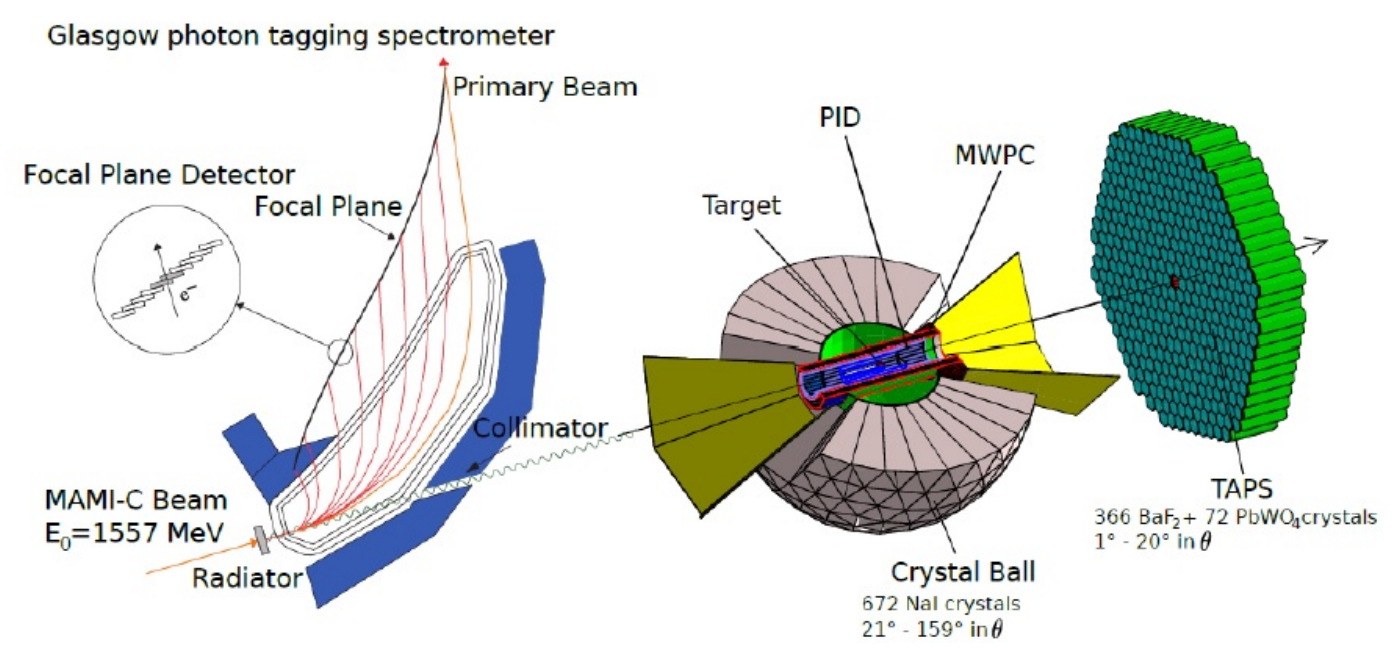

Figure 1: Schematic view of the A2 experimental setup.

\section{Selected Results}

\subsection{Single $\pi^{0}$ Photoproduction}

The beam-target asymmetry $F$ for the $\gamma p \rightarrow p \pi^{0}$ reaction was measured for the first time and new high precision data for the target asymmetry $T$ were obtained at photon energies from 425 to $1445 \mathrm{MeV}$ [15]. These asymmetries were expanded in terms of associated Legendre functions and a rather rapid change of the polynomial coefficients in the second and the third resonance regions, due to the presence of interference terms between selected resonances, was observed.

Also the double polarisation observables $G$ and $E$ were measured over a wide beam energy range and for the entire angular range $1 \leq \cos \theta_{\pi}^{c m} \leq 1$. Figures 2 and 3 show a part of the preliminary results available. They are compared to the published data from the CBELSA collaboration 
(when available) both for the $G$ observable [16] and for E [17] and to different partial wave analyses. The agreement with the existing data show the high quality of these new results. There is also a good agreement with the different PWA models, especially with the BnGa2014-02 PWA solution.

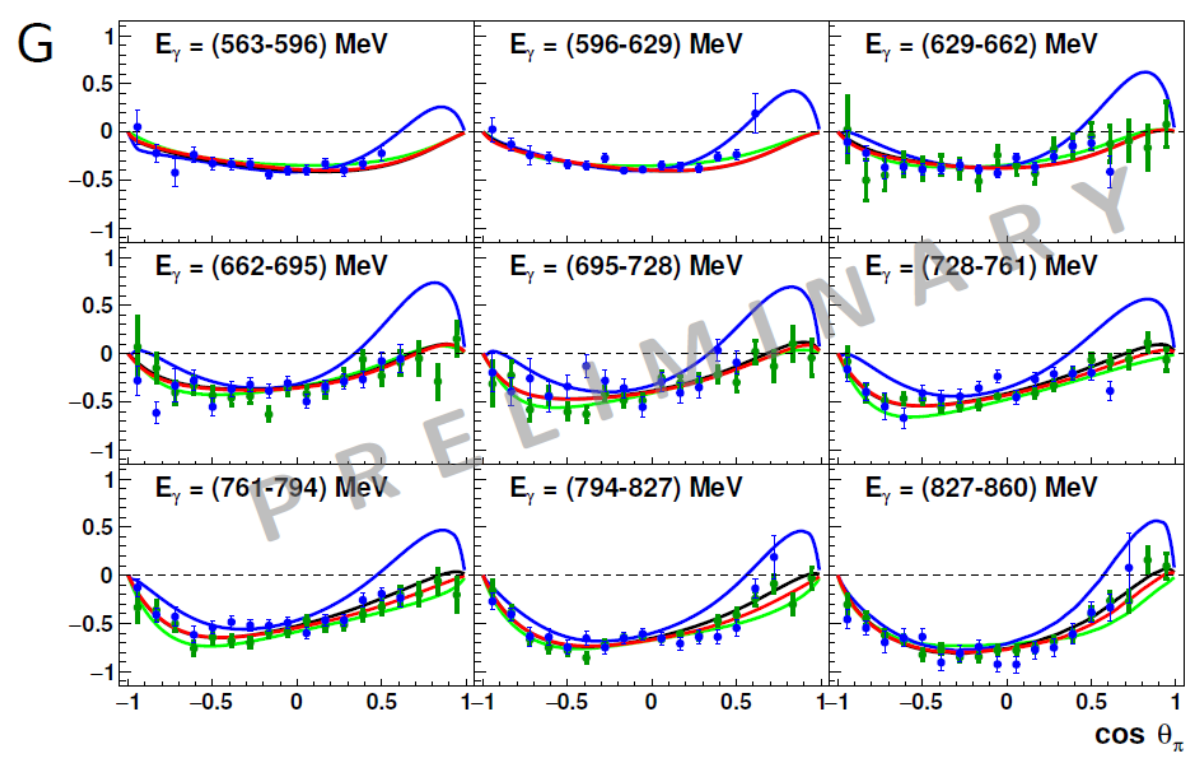

Figure 2: Preliminary results for $G$ in $\pi^{0}$-photoproduction for some selected energy bins, from $560 \mathrm{MeV}$ to $860 \mathrm{MeV}$. The experimental results from A2 (blue circles) are compared to the CBELSA data (green circles) [16], to PWA fits BnGa-2014-02 [18] (red line) and BnGa-2014-01 (black line) and PWA predictions from MAID2007 [19] (green line) and SAID-CM12 [20] (blue line).

As a general remark, the comparison of our data to results of recent model calculations and partial-wave analyses supports the observation that partial-wave amplitudes extracted in different approaches start to converge to model-independent values [21]. This is a success of the huge effort at ELSA, GRAAL, JLab, and MAMI to measure high precision polarization-dependent cross sections and asymmetries. The unique extraction of model-independent partial-wave amplitudes is an important prerequisite for the detailed understanding of the interplay between background and resonance contributions and the precise determination of excited baryon properties.

\subsection{Compton Scattering}

The $\Sigma_{2 x}$ double-spin asymmetry has been measured for the first time by the A2@MAMI collaboration [22]. This allowed the first experimental evaluation of the spin polarizability values.

The published data in the energy range of $\mathrm{E}_{\gamma}=273-303 \mathrm{MeV}$ are given in Fig. 4(a). The curves shown here are from the HDPV dispersion code [23] using different values for $\gamma_{E 1 E 1}$ that are differentiated by color, while $\alpha_{E 1}$ and $\beta_{M 1}$ are assigned using the PDG values [6], $\gamma_{0}=1.0$, $\gamma_{\pi}=8.0$ and $\gamma_{M 1 M 1}=2.9$. The width of each band is generated by allowing $\alpha_{E 1}, \beta_{M 1}, \gamma_{0}$, and $\gamma_{\pi}$, to vary within their experimental errors. This figure then gives a rough idea of the relevant sensitivity of this asymmetry to $\gamma_{E 1 E 1}$. Using just this observable, the values of $\gamma_{E 1 E 1}=4.6 \pm 1.6$ and $\gamma_{M 1 M 1}=7 \pm 11$ were extracted [22]. The value for $\gamma_{M 1 M 1}$ is poorly known due to the low sensitivity of $\Sigma_{2 x}$ to this observable, as also predicted by the HDPV model. 


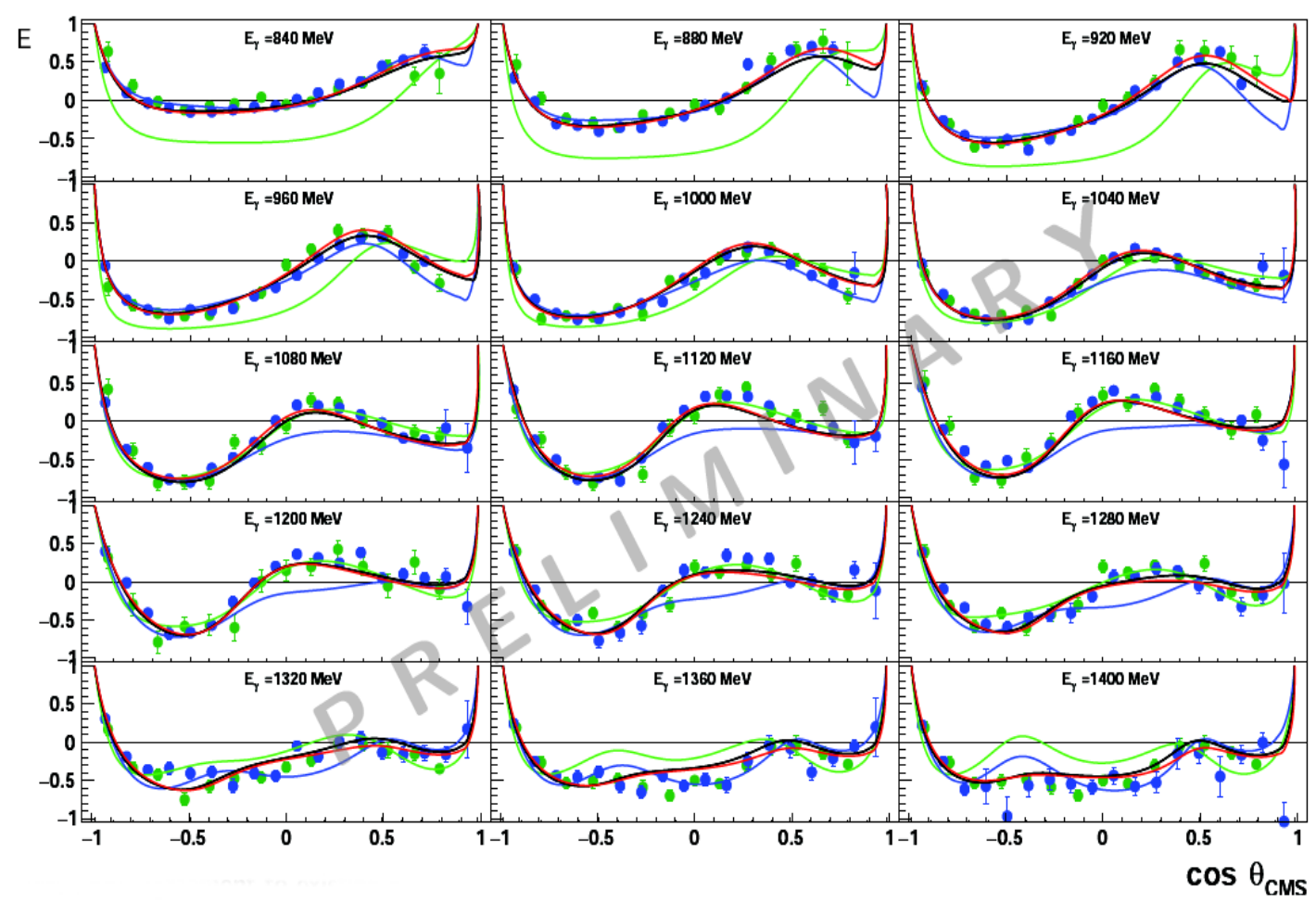

Figure 3: Preliminary results for $E$ in $\pi^{0}$-photoproduction for some selected energy bins, from $840 \mathrm{MeV}$ to $1400 \mathrm{MeV}$. The experimental results from A2 (blue circles) are compared to the CBELSA data (green circles) [17]. Curves as in the previous figure.

The $\gamma_{M 1 M 1}$ value can instead be much better constrained by measuring the $\Sigma_{2 z}$ observable. These data were recently taken by the A2@MAMI collaboration and preliminary results, which only include half of the available statistics, are shown in Fig. 4(b). This figure was constructed in a similar way as the previous one, except for varying $\gamma_{M 1 M 1}$ and keeping $\gamma_{M 1 M 1}$ fixed instead. From these preliminary values one can at least observe both reasonable agreement with the curves as well as indications of reasonably high constraining power of these data.

Data for unpolarized cross section and for the $\Sigma_{3}$ asymmetry have also been recently taken with improvements in both the tagging system and the linear beam polarization stability. These data will provide both a relevant reduction in the existing $\Sigma_{3}$ asymmetry experimental errors [24] and a set of unpolarised differential cross section data, that together will also enable a single extraction of $\alpha_{E 1}$ and $\beta_{M 1}$ at the level of the PDG errors. The combined used of all the different measured observables will then give a very significantly improvement to our experimental knowledge of all the polarizability values.

\section{Conclusions}

The experimental program carried out by the A2@MAMI collaboration has included experiments on transversely and longitudinally polarized targets with a circularly polarized photon beam, as well as on an unpolarized hydrogen target with a linearly polarized beam to measure a wide range on rections both on the proton and on the neutron. 

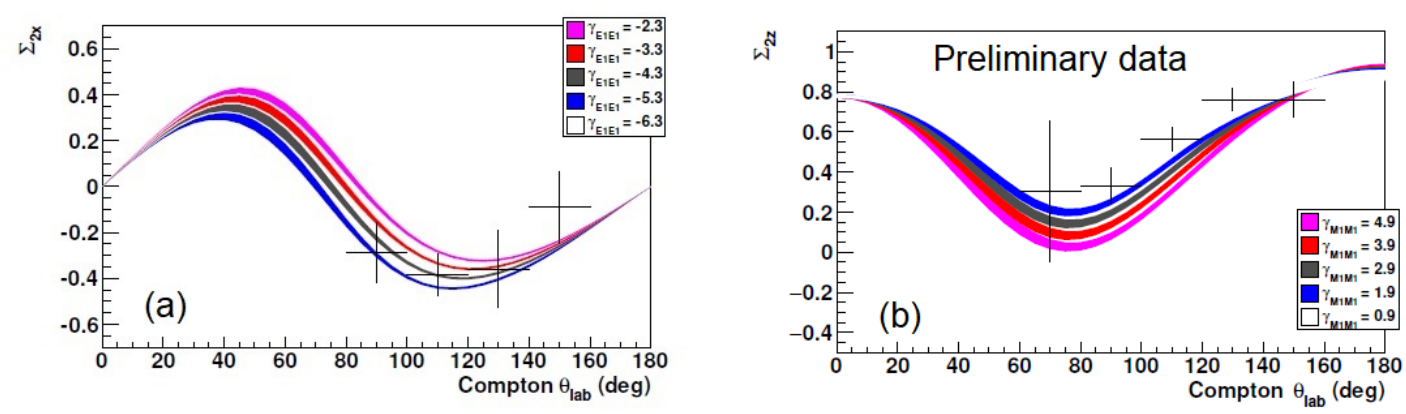

Figure 4: (a) $\Sigma_{2 x}$ for $\mathrm{E}_{\gamma}=273-303 \mathrm{MeV}$ [22]. The theoretical curves are from the HDPV dispersion model [23] using different values for $\gamma_{E 1 E 1}$. (b) $\Sigma_{2 z}$ for $\mathrm{E}_{\gamma}=273-303 \mathrm{MeV}$ (preliminary data). Similar to (a), but now $\gamma_{M 1 M 1}$ is allowed to vary, while one fixes $\gamma_{E 1 E 1}=-4.3$.

A part of the data has already been published and many of the remaining analyses of are almost completed. From all these new data, an accurate evaluation of the baryon resonance properties and a precise extraction the proton polarizability values can be expected.

\section{References}

[1] G. Chew et al., Relativistic Dispersion Relation Approach to Photomeson Production, Phys. Rev. 106 (1957) 1345.

[2] W.-T. Chiang and F. Tabakin, Completeness rules for spin observables in pseudoscalar meson photoproduction, Phys. Rev. C 55 (1997) 2054.

[3] I. Barker, A. Donnachie and J. Storrow, Complete experiments in pseudoscalar photoproduction, Nucl Phys. B 95 (1975) 347.

[4] R. Edwards et al., Excited state baryon spectroscopy from lattice QCD , Phys. Rev. D 84 (2011) 074508 .

[5] G. Huber and C. Collicott, The Nucleon Polarizability Program at MAMI-A2, in Proceedings of 12th Conference on the Intersections of Nuclear and Particle Physics (CIPANP 2015), 2015, nucl-ex/1508.07919.

[6] Particle Data Group collaboration, C. Patrignani et al., Review of Particle Physics, Chin. Phys. C 40 (2016) 100001.

[7] GDH collaboration, J. Ahrens et al., First measurement of the Gerasimov-Drell-Hearn integral for ${ }^{1} \mathrm{H}$ from 200 to $800 \mathrm{MeV}$, Phys. Rev. Lett. 87 (2001) 022003.

[8] GDH collaboration, H. Dutz et al., Experimental Check of the Gerasimov-Drell-Hearn Sum Rule for ${ }^{1}$ H , Phys. Rev. Lett. 91 (2003) 192001.

[9] M. Camen et al., Backward spin polarizability $\gamma_{\pi}$ of the proton, Phys. Rev. C 65 (2002) 032202.

[10] D. Goertz, Polarized target material developments at Bonn and Bochum , in Proc. XVIth International Workshop in Polarized Sources, Targets, and Polarimetry (PSTP2015), PoS(PSTP2015), p. 009, 2016.

[11] D. Crabb and W. Meyer, Solid polarized targets for nuclar and particle physics experiments, Annu. Rev. Nucl. Part. Sci. 47 (1997) 67.

[12] A. Starostin et al., Measurement of $K \vec{p} \rightarrow \eta \Lambda$ near threshold, Phys. Rev. $C 64$ (2001) 055205. 
[13] R. Novotny, The BaF 2 photon spectrometer TAPS , IEEE TRans. Nucl. Sci. 38 (1991) 379.

[14] A2 collaboration, C. M. Tarbert et al., Neutron Skin of 208Pb from Coherent Pion Photoproduction, Phys. Rev. Lett. 112 (2014) 242502.

[15] A2 collaboration, J. Annand et al., T and F asymmetries in $\pi^{0}$ photoproduction on the proton, Phys. Rev. C 93 (2016) 055209.

[16] A. Thiel et al., Well-established nucleon resonances revisited by double-polarization measurements. , Phys. Rev. C 65 (2002) 032202.

[17] M. Gottschall et al., First Measurement of the Helicity Asymmetry for $\gamma p \rightarrow p \pi^{0}$ in the Resonance Region, Phys. Rev. Lett. 112 (2014) 012003.

[18] E. Gutz et al., High statistics study of the reaction $\gamma p \rightarrow p \pi^{0} \eta$, Eur. Phys. J. A 50 (2014) 74.

[19] D. Drechsel, S. Kamalov and L. Tiator, Unitary isobar model-MAID2007, Eur. Phys. J. A 34 (2007) 69.

[20] R. Workman et al., Unified Chew-Mandelstam SAID analysis of pion photoproduction data, Phys. Rev. $C 86$ (2012) 015202.

[21] A. Anisovich et al., The impact of new polarization data from Bonn, Mainz and Jefferson Laboratory on $\gamma p \rightarrow \pi N$ multipoles, Eur. Phys. J. A 52 (2016) 2284.

[22] A2 collaboration, P. P. Martel et al., Measurements of Double-Polarized Compton Scattering Asymmetries and Extraction of the Proton Spin Polarizabilities, Phys. Rev. Lett. 114 (2015) 112501.

[23] B. Pasquini et al., Proton spin polarizabilities from polarized Compton scattering, Phys. Rev. C 76 (2007) 015203.

[24] A2 collaboration, V. Sokhoyan et al., Determination of the scalar polarizabilities of the proton using beam asymmetry $\Sigma_{3}$ in Compton scattering, Eur. Phys. J. A 53 (2017) 14. 